\title{
Using a novel multi-variable grey model to forecast the electricity consumption of Shandong Province in China
}

\author{
Lifeng $\mathrm{Wu}^{* a}$, Xiaohui $\mathrm{Gao}^{a}$, Yanli Xiao ${ }^{* a}$, Yingjie Yang ${ }^{b}$, Xiangnan $\mathrm{Chen}^{a}$ \\ ${ }^{a}$ College of Economics and Management, Hebei University of Engineering, Handan, 056038, China \\ ${ }^{b}$ Centre for Computational Intelligence, De Montfort University, Leicester, LE1 9BH, UK
}

1 Abstract:

2

The electricity consumption forecasting problem is especially important for policy making in developing region. To properly formulate policies, it is necessary to have reliable forecasts. Electricity consumption forecasting is influenced by some factors, such as economic, population and so on. Considering all factors is a difficult task since it requires much detailed study in which many factors significantly influence on electricity forecasting whereas too many data are unavailable. Grey convex relational analysis is used to describe the relationship between the electricity consumption and its related factors. A novel multi-variable grey forecasting model which considered the total population is developed to forecast the electricity consumption in Shandong Province. The GMC $(1, \mathrm{~N})$ model with fractional order accumulation is optimized by changing the order number and the effectiveness of the first pair of original data by the model is proven. The results of practical numerical examples demonstrate that the model provides remarkable prediction performances compared with the traditional grey forecasting model. The forecasted results showed that the increase of electricity consumption will speed up in Shandong Province.

Keywords: electricity consumption forecasting; multi-variable grey model; population; fractional order accumulation

\section{Introduction}

Energy plays an important role in the course of economy development and social progress. Energy forecasting constitutes a vital part of energy policy of a country, especially for a developing country like China whose economy is in a stage of energy consumption structure adjustment [1]. This has motivated many researchers to focus on energy forecasting. Such as, Chai et al. used Bayesian combination model to forecast energy demand of China [2]. Ji predicted the petroleum

\footnotetext{
${ }^{0 *}$ Corresponding Author: E-mail address: wlf6666@126.com, 84536065@qq.com
} 
consumption in China by comparing three models [3]. Niu and Meng predicted annual electricity consumption in China [4]. Zhang et al. forecasted Chinese transport energy demand based on partial least square regression [5], Li et al. used the least squares support vector machine with a fruit fly optimization algorithm to predict the annual electricity load [6]. Zhu et al. provided a seasonal hybrid procedure for electricity demand forecasting in China [7]. Li et al. combined the adaptive grey model with intelligence computation to predict the short-term electricity consumption [8]. Hu and Jiang used a neural-network-based grey residual modification to forecast the energy demand [9]. He et al. proposed a hybrid model equipped with the minimum cycle decomposition concept for forecasting electrical load over a short term [10]. Ma and Liu put forward a novel time-delayed polynomial grey model to predict the natural gas consumption in China [11]. Tsai et al. confirmed that the modified grey model had a higher forecasting accuracy for renewable energy than the original grey model [12]. Xu et al. discussed the grey prediction model with the nonlinear optimized time response method for forecasting electricity consumption in China [13]. Grey Verhulst model and the nonlinear grey Bernoulli model have forecasted that the Chinese natural gas demand will reach 315 billion $\mathrm{m}^{3}$ by 2020 [14]. Turkey's sectoral energy demand is forecasted by using a fuzzy grey regression model [15]. The electricity demand across different countries is forecasted 24 months in advance [16]. The real monthly electricity consumption and macroeconomic data from China have been studied to verify the effectiveness of the vector error correction model [17]. A comprehensive review and summarization of decomposition based approach for the electricity demand forecasting is conducted [18]. The week day/weekend/holiday consumption profiles to infer the proportion of industrial and domestic electricity consumption is discussed [19]. The bottomup approach is used to evaluate the trajectory of long term annual electricity consumption of a sector of the Brazilian industry up to 2050 considering energy efficiency scenarios [20]. Two deep recurrent neural network models are proposed for electricity forecasting [21].

The conventional energy consumption prediction models can be roughly divided into three types: regression model, intelligence computational technologies and time series method. However, the prediction accuracies of the time series method and regression method rely on the distribution of the original series as well as a large amount of observed data. The successes of intelligence 
computational technology needs a large amount of training data. In many practical situations, because of limitation for time and cost, it is very difficult to obtain the complete information from the analyzed system. In order to accurately analyze and predict the uncertain systems, many studies on energy consumption forecasting using grey models and improved grey models have been reported. Kumar and Jain clearly demonstrated that the time series models (grey-Markov model, grey-model with rolling mechanism and singular spectrum analysis) have enormous potential for forecasting energy consumption [22]. Zhou presented a trigonometric grey prediction approach for forecasting electricity demand by combining the traditional grey model with the trigonometric residual modification technique [23]. Lee and Tong developed an improved grey forecasting model that combined residual modification with genetic programming sign estimation [24]. Diyar and Mehmet proposed grey rolling mechanism approach to predict the Turkey's total and industrial electricity consumption [25]. Pao and Tsai compared the forecasting ability of the grey model with that of the autoregressive integrated moving average model over the out-of-sample period between 2002 and 2007 [26]. Pao et al. proposed a numerical iterative method to optimize the parameters of the nonlinear grey Bernoulli model [27]. Li et al. applied the cubic spline function and Taylor approximation method to optimize the grey model for achieving a high power system load forecasting accuracy [28]. An overview of energy demand grey forecasting methods published in 2005-2015 is given [29]. However, these models are all first-order grey models with one variable and only contain the information relating to the predicted series during modelling. Therefore, these models have significant limitations [30].

The multi-variable grey forecasting model is represented by $\operatorname{GM}(1, N), \mathrm{GM}(1, \mathrm{~N})$ is composed of a system characteristic sequence (or dependent variable sequence) and (N-1) related factor sequences (or independent variable sequences). The modeling process takes full account of the effect of the relevant factors on the system change, and it is a typical causal forecasting model. It can make full use of the information contained in the associated series. In the view of the available additional information, $\operatorname{GM}(1, n)$ is likely to show higher forecasting accuracy than GM(1,1) [31]. Therefore, in this paper, a new multi-variable grey model is used to forecast energy consumption. The main contributions of this paper are summarized below. 1) The first pair of original data 
by the model is effectiveness. Practical examples demonstrate that the model provides remarkable predictive performance. 2) To obtain more valuable data, the grey convex relational method is applied to identify the key factor associated with electricity consumption. 3) A multi-variable grey forecasting model that considered total population is implemented to forecast the electricity consumption in Shandong Province. 4) The proposed forecasting method can effectively predict the future electricity consumption and outlook the consumption trend in Shandong province.

The rest of the paper proceeds as follows. Section 2 is a compendium of annual electricity consumption in Shandong Province, China. A novel grey model is presented in Section 3. The electricity consumption of Shandong Province in China is predicted in Section 4. Some conclusions and discussion are given in the final Section.

\section{Annual electricity consumption in Shandong Province}

Because electricity is a major energy source, the electricity consumption has been a major measurement to indicate the level of region development. It is necessary to forecast electricity consumption of a region. Shandong Province is a typical representative of China in the economic growth and energy consumption. The coastline of Shandong is about $3300 \mathrm{~km}$ in length. Approximately $32 \%$ of the population reside in the coastal zone. The coastal zones are the most rapidly developed areas of Shandong. The State Council has officially approved the "Development Plan of High-efficient Ecological and Economic Zone of Yellow River Delta". As a beginning, the development of Yellow River Delta area has been upgraded to the national strategy and has become an important component of harmonious development of the regions. Shandong Province is situated in the northern China plain, at the eastern coastline and lower reaches of Yellow River. Its

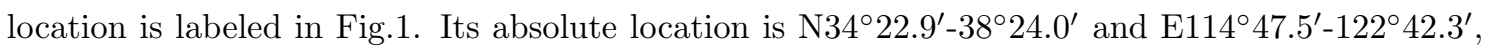
whose total area is 156.7 thousand $\mathrm{km}^{2}$. It is one of the largest economies and most populated Provinces in China. In 2002, the gross domestic product (GDP) of this province ranked the third in China, accounting for about one-tenth of the total national GDP, and the population ranked the second. In 2008, it ranked the second in GDP but the first in total energy consumption in China. Meanwhile, the economic structure and economic development pattern of Shandong Province has 
a similarity to the nation. Therefore, it is important to forecast the annual electricity consumption of Shandong Province.

fig1

In Shandong Province, electricity consumption is increasing rapidly due to the increasing population size, creating continuous pressures for better living standards and emphasis on large-scale industrialization. GDP growth has also played a driving role. Therefore, many factors closely related to the electricity consumption are selected. They are GDP, urban per capita disposable income, total population, industrial output value and fixed assets investment. These factors have greatly affected electricity consumption. Because the energy policy in Shandong Province is relatively steady since 2003, the selected time period is from 2003 to 2015. All the annual data are collected from the Shandong Statistical Yearbook (http://www.stats-sd.gov.cn/col/col211/index.html). All variables are described in Table 1. As shown in Table 1, which reflects the development trends of electricity consumption and its related indicators.

Table 1 The actual value of each factor in Shandong Province

As the economic situation of Shandong Province has improved recently. Its electricity consumption grew by $17.5 \%$ in 2004 and $4.38 \%$ in 2012 , which were both lower than GDP growth. From 2004 to 2012, the urban per capita disposable income increased by around $13 \%$. The total population size grew slightly. The industrial output value has continuously increased from 1989 to 12229 billion Yuan. the fixed assets investment has increased from 5328 to 3032 billion Yuan. In-sample data (2003-2012) is used to estimate model parameters and out-of-sample data (20132015 ) is used to evaluate the forecasting performance in order to investigate the feasibility of the novel model.

\section{The $\operatorname{GMC}(1, \mathrm{~N})$ model with fractional order accumulation}

The grey prediction model with convolution integral $(\mathrm{GMC}(1, \mathrm{~N}))$, proposed by Tien [32], is a new model to improve the traditional $\operatorname{GM}(1, N)$. The values modelled by $\operatorname{GMC}(1, \mathrm{~N})$ are the exact solution of the traditional $\operatorname{GM}(1, \mathrm{~N})$ model. The $\operatorname{GMC}(1, \mathrm{~N})$ model has successfully been applied in 


\subsection{The modeling method of the $\operatorname{GMC}(1, N)$ with fractional order accumulation}

Definition 1 It is assumed that $X_{1}^{(0)}=\left\{x_{1}^{(0)}(1), x_{1}^{(0)}(2), \cdots, x_{1}^{(0)}(r)\right\}$ is the sequence of system characteristic data, and $X_{i}^{(0)}=\left\{x_{i}^{(0)}(1), x_{i}^{(0)}(2), \cdots, x_{i}^{(0)}(r)\right\}(i=2,3, \cdots, \mathrm{N})$ are the sequences of the relevant factors. Then

$$
\frac{d x_{1}^{\left(\frac{p}{q}\right)}(t)}{d t}+b_{1} x_{1}^{\left(\frac{p}{q}\right)}(t)=b_{2} x_{2}^{\left(\frac{p}{q}\right)}(t)+b_{3} x_{3}^{\left(\frac{p}{q}\right)}(t)+\cdots+b_{n} x_{n}^{\left(\frac{p}{q}\right)}(t)+u
$$

is the $\operatorname{GMC}^{\frac{p}{q}}(1, \mathrm{~N})$ model, where $x_{i}^{\left(\frac{p}{q}\right)}(k)$ is the $\frac{p}{q}$ order accumulation of $x_{i}^{(0)}(k), x_{i}^{\left(\frac{p}{q}\right)}(k)=$ $\sum_{j=1}^{k}\left(\begin{array}{c}k-j+r-1 \\ k-j\end{array}\right) x_{i}^{(0)}(j), k=1,2, \cdots, r[35]$. It is the traditional $\operatorname{GMC}(1, \mathrm{~N})$ model when $\frac{p}{q}=1$. Using the ordinary least squares, the parameter of $\operatorname{GMC}^{\frac{p}{q}}(1, \mathrm{~N})$ model are estimated:

$$
\left[\hat{b}_{1}, \hat{b}_{2}, \cdots, \hat{b}_{n}, \hat{u}\right]^{T}=\left(B^{T} B\right)^{-1} B^{T} Y
$$

where

$$
Y=\left[\begin{array}{c}
x_{1}^{\left(\frac{p}{q}\right)}(2)-x_{1}^{\left(\frac{p}{q}\right)}(1) \\
x_{1}^{\left(\frac{p}{q}\right)}(3)-x_{1}^{\left(\frac{p}{q}\right)}(2) \\
\vdots \\
x_{1}^{\left(\frac{p}{q}\right)}(n)-x_{1}^{\left(\frac{p}{q}\right)}(n-1)
\end{array}\right]
$$

$$
B=\left[\begin{array}{ccccc}
-0.5\left(x_{1}^{\left(\frac{p}{q}\right)}(2)+x_{1}^{\left(\frac{p}{q}\right)}(1)\right) & 0.5\left(x_{2}^{\left(\frac{p}{q}\right)}(2)+x_{2}^{\left(\frac{p}{q}\right)}(1)\right) & \cdots & 0.5\left(x_{n}^{\left(\frac{p}{q}\right)}(2)+x_{n}^{\left(\frac{p}{q}\right)}(1)\right) & 1 \\
-0.5\left(x_{1}^{\left(\frac{p}{q}\right)}(3)+x_{1}^{\left(\frac{p}{q}\right)}(2)\right) & 0.5\left(x_{2}^{\left(\frac{p}{q}\right)}(3)+x_{2}^{\left(\frac{p}{q}\right)}(2)\right) & \cdots & 0.5\left(x_{n}^{\left(\frac{p}{q}\right)}(3)+x_{n}^{\left(\frac{p}{q}\right)}(2)\right) & 1 \\
\vdots & \vdots & \vdots & \vdots \\
-0.5\left(x_{1}^{\left(\frac{p}{q}\right)}(r-1)+x_{1}^{\left(\frac{p}{q}\right)}(r)\right) & 0.5\left(x_{2}^{\left(\frac{p}{q}\right)}(r-1)+x_{2}^{\left(\frac{p}{q}\right)}(r)\right) & \cdots & 0.5\left(x_{n}^{\left(\frac{p}{q}\right)}(r-1)+x_{n}^{\left(\frac{p}{q}\right)}(r)\right) & 1
\end{array}\right]
$$

Set $\hat{x}_{1}^{(0)}(1)=x_{1}^{(0)}(1)$, when $t \geq 2$, the convolution integral of the right hand of Eq.(1) can also be discretised as:

$$
x_{1}^{\left(\frac{p}{q}\right)}(t)=x_{1}^{(0)}(1) e^{-b_{1}(t-1)}+\sum_{\tau=2}^{t}\left\{e^{-b_{1}(t-\tau+0.5)} \frac{f(\tau)+f(\tau-1)}{2}\right\}
$$

where

$$
f(t)=b_{2} x_{2}^{\left(\frac{p}{q}\right)}(t)+b_{3} x_{3}^{\left(\frac{p}{q}\right)}(t)+\cdots+b_{n} x_{n}^{\left(\frac{p}{q}\right)}(t)+u
$$


Then the predicted value is given by $\frac{p}{q}\left(0 \leq \frac{p}{q} \leq 1\right)$ order inverse accumulated generating operator

$$
\hat{X}^{(0)}=\alpha^{(1)} \hat{X}^{\left(1-\frac{p}{q}\right)}(k)=\left\{\alpha^{(1)} \hat{x}^{\left(1-\frac{p}{q}\right)}(1), \alpha^{(1)} \hat{x}^{\left(1-\frac{p}{q}\right)}(2), \cdots, \alpha^{(1)} \hat{x}^{\left(1-\frac{p}{q}\right)}(n)\right\} .
$$

140

\subsection{The effectiveness of the first pair of original data by $\operatorname{GMC}^{\frac{p}{q}}(1, N)$}

The modeling values and forecasting values are proved to be independent of the first pair of original data by $\operatorname{GMC}(1, N)[36]$. In this paper, The modeling values and forecasting values are proved to be dependent of the first pair of original data by $\operatorname{GMC}^{\frac{p}{q}}(1, \mathrm{~N})$ when $\frac{p}{q}$ is not an integral number. Without losing generality, we can take factor number $N$ as 2 for convenience, a real case is from Reference $[33,37]$. The procedures of $\operatorname{GMC}^{\frac{p}{q}}(1, \mathrm{~N})$ can be summarized as follows:

Step 1: For the sequence $x_{1}^{(0)}=\{897,897,890,876,848\}$, according to Definition 1, we have

$$
\begin{gathered}
x_{1}^{(1.03)}(1)=x_{1}^{(0)}(1)=897, \\
x_{1}^{(1.03)}(2)=897 \times 1.03+897=1820.9, \\
x_{1}^{(1.03)}(3)=897 \times \frac{2.03 \times 1.03}{2}+897 \times 1.03+890=2751.7, \\
x_{1}^{(1.03)}(4)=897 \times \frac{3.03 \times 2.03 \times 1.03}{3 \times 2}+897 \times \frac{2.03 \times 1.03}{2}+890 \times 1.03+876=3677.6, \\
x_{1}^{(1.03)}(5)=897 \times \frac{4.03 \times 3.03 \times 2.03 \times 1.03}{4 \times 3 \times 2}+897 \times \frac{3.03 \times 2.03 \times 1.03}{3 \times 2}+890 \times \frac{2.03 \times 1.03}{2}+876 \times 1.03+848=4582.1,
\end{gathered}
$$

Therefore, 1.03 order accumulation sequence is $x_{1}^{(1.03)}=\{897,1820.9,2751.7,3677.6,4582.1\}$.

Step 2: Similarly, for the sequence $x_{2}^{(0)}=\{514,495,444,401,352\}, 1.03$ order accumulation sequence is $x_{2}^{(1.03)}=\{514,1024.4,1491.2,1918.6,2298.7\}$. The parameters $B$ and $Y$ in Eq.(2) can be obtained by employing the first five pairs as

$$
B=\left[\begin{array}{ccc}
-1358.96 & 769.21 & 1 \\
-2286.29 & 1257.82 & 1 \\
-3214.65 & 1704.88 & 1 \\
-4129.87 & 2108.62 & 1
\end{array}\right], Y=\left[\begin{array}{l}
923.91 \\
930.77 \\
925.94 \\
904.51
\end{array}\right]
$$

Step 3: Substituting the $B$ and $Y$ into Eq.(2), we have the parameters $b_{1}, b_{2}, u$. Then, $\operatorname{GMC}^{1.03}(1,2)$ can be represented as

$$
\frac{d x_{1}^{(1.03)}(t)}{d t}+0.1798 x_{1}^{(1.03)}(t)=0.3579 x_{2}^{(1.03)}(t)+892.57, t=1,2, \cdots .
$$


Step 4: The discrete function $f(t)$ in Eq.(6) for the $\mathrm{GMC}^{1.03}(1,2)$ is obtained to be listed in Table 2 .

Table 2 The discrete function $f(t)$ in (5) for periods 1 to 10

Step 5: The 1.03 order accumulated generating operation values $\hat{x}_{1}^{(1.03)}$ can be obtained by employing the Eq.(5):

$$
\hat{x}_{1}^{(1.03)}=\{897,1816.9,2745.3,3667.2,4569.5,5438.1,6265.0,7047.3,7781.0,8465.8\} .
$$

The 2 order accumulated generating operation values $\hat{x}_{1}^{(2)}$ are

$\hat{x}_{1}^{(2)}=\hat{x}_{1}^{(1.03)(0.97)}=\{897,2687.0,5364.7,8914.6,13310.4,18513.9,24480.4,31163.4,38513.6,46482.2\}$.

The 1 order accumulated generating operation values $\hat{x}_{1}^{(1)}$ are

$$
\hat{x}_{1}^{(1)}=\{897,1790.0,2677.7,3549.8,4395.8,5203.5,5966.4,6683.0,7350.2,7968.6\} .
$$

The predicted values $\hat{x}_{1}^{(0)}$ are $\hat{x}_{1}^{(0)}=\{897,893.0,887.7,872.1,846.0,807.7,762.9,716.6,667.2,618.4\}$, which are listed in Table 3.

If the first pair of original data has changed, for example, the original data are $\hat{x}_{1}^{(0)}=$ $\{797,897,890$,

$876,848\}, x_{2}^{(0)}=\{564,495,444,401,352\}$. Following steps 1-5, the $\operatorname{GMC}^{1.03}(1,2)$ can be represented as

$$
\frac{d x_{1}^{(1.03)}(t)}{d t}+0.1838 x_{1}^{(1.03)}(t)=0.3668 x_{2}^{(1.03)}(t)+851.07, t=1,2, \cdots
$$

Then, the predicted values are listed in Table 3. Eq.(8) and Eq.(9) are different, because the first pair of original data are different. Therefore, when $\frac{p}{q}$ is not an integral number, the first pair of original data by $\operatorname{GMC}^{\frac{p}{q}}(1, \mathrm{~N})$ is effectiveness, because the parameters $\left(b_{1}\right.$ and $\left.b_{2}\right)$ of $\operatorname{GMC}^{\frac{p}{q}}(1, \mathrm{~N})$ are dependent of the arbitrary numbers added to $x_{1}^{(0)}$ and $x_{2}^{(0)}$ respectively. When $\frac{p}{q}$ is an integral number, adding the arbitrary numbers to $x_{1}^{(0)}$ and $x_{2}^{(0)}$ indicate a translation in $x_{1}^{(0)}$ and $x_{2}^{(0)}$ respectively.

Table 3 The results of different grey models

\subsection{Validation of the $\operatorname{GMC}^{\frac{p}{q}}(1, \mathrm{~N})$ model}

In this section, the advantages of the $\operatorname{GMC}^{\frac{p}{q}}(1, \mathrm{~N})$ over the existing $\operatorname{GMC}(1, \mathrm{~N})$ are demonstrated by three cases. The root mean squared percentage error (RMSPE) for the priori-sample 
period (RMSPEPR) and post-sample periods (RMSPEPO) suggested by Tien [33] are used to evaluate the precision. RMSPEPR and RMSPEPO are defined as

$$
\text { RMSPEPR }=100 \% \sqrt{\frac{1}{r} \sum_{t=1}^{r}\left(\frac{\hat{x}_{1}^{(0)}(t)-x_{1}^{(0)}(t)}{x_{1}^{(0)}(t)}\right)^{2}}
$$

and

$$
\text { RMSPEPO }=100 \% \sqrt{\frac{1}{r f} \sum_{t=r+1}^{r+r f}\left(\frac{\hat{x}_{1}^{(0)}(t)-x_{1}^{(0)}(t)}{x_{1}^{(0)}(t)}\right)^{2}}
$$

$r f$ is the number of entries to be forecasted.

\section{Example 1: Predicting the gross industrial output value}

To verify the forecasting accuracy of the $\operatorname{GMC}^{\frac{p}{q}}(1, \mathrm{~N})$. This example is from Reference [37]. The actual values are listed in Table 4 . The first seven groups data are used for building the model and the last six groups data are used for testing.

Table 4 Raw data used in example 1

Applying the $\operatorname{GMC}^{\frac{p}{q}}(1,3)$ model by Eq.(1)-(7), we have the parameters of $b_{1}, b_{2}, b_{3}, u$. Then, $\operatorname{GMC}^{\frac{p}{q}}(1,3)$ can be represented as:

$$
\frac{d x_{1}^{(1.6)}(t)}{d t}+0.6204 x_{1}^{(1.6)}(t)=5.3611 x_{2}^{(1.6)}(t)-2.6388 x_{3}^{(1.6)}(t)+72036.7456,
$$

\section{The predicted values of $\mathrm{GMC}^{1.6}(1,3)$ are listed in Table 5. The RMSPEPR and RMSPEPO} of four models are shown in Table 6, and also plotted in Fig. 2.

Table 5 The predicted values in $\operatorname{GMC}^{1.6}(1,3)$

Table 6 The RMSPEPR and RMSPEPO of four models

Fig. 2

The RMSPEPR and RMSPEPO of the $\operatorname{GMC}^{\frac{p}{q}}(1,3)$ model are as small as $3.77 \%$ and $3.48 \%$, respectively. The results indicate that the $\operatorname{GMC}^{\frac{p}{q}}(1,3)$ model outperforms the other models in this example.

\section{Example 2: Indirect measurement of the tensile strength of a material}

This example is from Reference [33, 37]. The first five pairs of observations are used to build the model and the last five pairs are used for testing. The results of $\operatorname{GMC}^{1.03}(1,2)$ are given in Table 3. The RMSPEPR and RMSPEPO of different models are shown in Table 7, and are plotted in Fig. 3. 
Table 7 The RMSPEPR and RMSPEPO of four models

Fig. 3

The RMSPEPR of these four models are very close to each other. Among these four models, the RMSPEPO of $\operatorname{GMC}^{1.03}(1,2)$ is the smallest. This indicates that $\operatorname{GMC}^{\frac{p}{q}}(1,2)$ can predict the tensile strength.

\section{Example 3: Economic output of Zhejiang Province in China}

This example is from Reference [38]. The first six pairs of observations are used for building the model and the last one pairs are used for testing. The results of $\mathrm{GMC}^{0.68}(1,2)$ are given in Table 8. The RMSPEPR and RMSPEPO of different models are shown in Table 9 and also plotted in Fig. 4.

Table 8 The results of different model

Table 9 The RMSPEPR and RMSPEPO of four models

Fig. 4

Among these three models, the RMSPEPR and RMSPEPO of $\operatorname{GMC}^{0.68}(1,2)$ are the smallest. It indicates that $\operatorname{GMC}^{\frac{p}{q}}(1,2)$ is remarkably superior to the conventional $\operatorname{GM}(1,2)$ owing to its higher forecast accuracy.

When $\frac{p}{q}$ is fractional rather than integral, considering the availability of additional information, $\operatorname{GMC}^{\frac{p}{q}}(1, \mathrm{~N})$ can make full use of the all data information, including the first pair of original data. In the view of the modelling precision, $\operatorname{GMC}^{\frac{p}{q}}(1, \mathrm{~N})$ has more degrees of freedom and better performance compared with the traditional one-order grey model. When $\frac{p}{q}$ is an integral, in the view of the available additional information, $\operatorname{GMC}^{\frac{p}{q}}(1, N)$ can not use the first pair of original data.

\section{Forecasting Shandong's electricity consumption by the $\operatorname{GMC}^{\frac{p}{q}}(1, \mathrm{~N})$ model}

\subsection{Selection of affecting factors via grey relational analysis}

Grey relational analysis, proposed by Deng, can be used to describe the relationships between the reference series and comparison series [39]. The calculated relational extent is proportional to the similarity of the developing trends, i.e., the more similar are the developing trends, the 
greater is the relational extent. The magnitude order of the relational grade implies the influential degree order of corresponding factors. Considering the limitations of the existing grey relational analysis, grey convex relational analysis is used to describe the relationship between the electricity consumption and its related factors.

Within the period 2003-2012, according to the method presented in [39], the order of grey convex relational analysis $\left\{y_{i}\right\}$ were as follows:

$$
y_{3}>y_{1}>y_{2}>y_{4}>y_{5}
$$

where $y_{1}=0.9818(\mathrm{GDP}), y_{2}=0.9817$ (urban per capita disposable income), $y_{3}=0.9854$ (total population), $y_{4}=0.9761$ (industrial output value), $y_{5}=0.9619$ (fixed assets investment). The order means the degree of influence of each variable on the electricity consumption, which indicates that the fixed assets investment has little effect on the electricity consumption, while the total population has the greatest influence. So in the present study, total population represent the independent variables (or the factors that affects electricity consumption).

\subsection{Forecasting analysis}

In this section, particle swarm optimization (PSO) is used to find the optimal fractional order. PSO is a global optimization algorithm proposed by Kennedy and Eberhart in 1995, which was derived from the study of foraging behavior of birds [40]. Due to its simple concept and powerful global search ability, PSO has been effectively applied in many fields to identify the optimum solution, such as, multi-objective dynamic economic emission dispatch [41], tire mixing process scheduling [42], watershed management learning [43], and numerical function optimization [44]. For the learning strategy, Ye Wenxing presented a novel multi-swarm PSO with a dynamic learning strategy to improve its performance [45]. For the update mechanism, Mustafa Servet Kiran proposed PSO with a new update mechanism, which is used to predict the global optimum by extracting the features that reflect the evolutionary trend [46]. In this section, PSO is adopted to find the optimal order which produces the minimum RMSPEPR and RMSPEPO. The experiments is conducted in the MATLAB R2015b. The analyses of the case are described in detail below.

$x_{1}^{(0)}$ is the electricity consumption, and $x_{2}^{(0)}$ is the total population. The original sequences are as follows: $x_{1}^{(0)}=\{1439.39,1691.28, \cdots, 3635.26\}, x_{2}^{(0)}=\{9108,9163, \cdots, 9580\}$. Following step 
$1-5$, the $\operatorname{GMC}^{0.71}(1,2)$ is

$$
\frac{d x_{1}^{(0.71)}(t)}{d t}-0.051 x_{1}^{(0.71)}(t)=0.0009 x_{2}^{(0.71)}(t)+1159.8
$$

and $\operatorname{GMC}^{1}(1,2)$

$$
\frac{d x_{1}^{(1)}(t)}{d t}+0.0105 x_{1}^{(1)}(t)=0.0311 x_{2}^{(1)}(t)+1310.2
$$

The driving coefficient $\hat{b}_{2}$ of different $\operatorname{GMC}^{\frac{p}{q}}(1,2)$ models are positive, which indicates that an increase in population cause increase in electricity consumption. The results support the actual situation. Because more electricity will be consumed as the population size increases. To reduce the electricity consumption, we must tell the people to save the electricity resources and it is also necessary to control the total population, because the people is the active of the electricity consumption. As we all known, the second-child policy has been implemented in Shandong Province. The population growth will speed up. The electricity consumption of Shandong Province will increase so rapidly. Hence, on one hand, Shandong Province needs to guide the development of technologyintensive industries to realize energy conservation. It is necessary to promote the development of low energy-consuming industries, meanwhile to limit and improve the high energy-consuming industries. On the other hand, in the future, Shandong province should impose an accurate plan to avoid the binding effects of the market economy and guarantee adequate electricity provision.

The discretised form of $\operatorname{GMC}^{0.71}(1,2)$ is

$$
x_{1}^{(0.71)}(t)=1439.39 e^{0.051(t-1)}+\sum_{\tau=2}^{t}\left\{e^{0.051(t-\tau+0.5)} \frac{f(\tau)+f(\tau-1)}{2}\right\},
$$

where $f(t)=0.0009 x_{2}^{(0.71)}(t)+1159.8$.

The total population size from 2013 to 2015 is $\{9612,9747,9822\}$. Substituting the total population size from 2003 to 2015 into Eq.(15). Following the step 5 mentioned above, the predictive values of $\operatorname{GMC}^{0.71}(1,2)$ can be obtained. The modeling process of $\operatorname{GM}(1,1)$ and $\operatorname{GMC}(1,2)$ are the same as those in Reference [33]. Then the predictive values of three models are given in Table 10. The RMSPEPR and RMSPEPO of three models are also plotted in Fig. 5. As shown in Table 10, according to the RMSPEPR and RMSPEPO criteria, the GMC $(1,2)$ model is better than the traditional $\operatorname{GM}(1,1)$ model. The predictive results of $\operatorname{GMC}^{\frac{p}{q}}(1,2)$ are improved compared with traditional $\operatorname{GMC}(1,2)$ model. It can be concluded that $\operatorname{GMC}^{\frac{p}{q}}(1,2)$ is able to simulate and predict 
the electricity consumption. The $\operatorname{GMC}^{\frac{p}{q}}(1, \mathrm{~N})$ may be more suitable for the electricity consumption system, because this model considers multiple variables, and $\frac{p}{q}$ can change along with the memory process of multiple variables.

Fig. 5

Table 10 The predictive values of different models

\section{Conclusion and discussion}

From the perspective of application, in Shandong Province, the total population is the key factor on the electricity consumption, as determined by grey convex relational analysis. The $\operatorname{GMC}^{\frac{p}{q}}(1, \mathrm{~N})$ model for electricity consumption forecasting is developed by considering the total population. The results show that the proposed approach outperforms the traditional $\operatorname{GMC}(1, \mathrm{~N})$ model, not only in its prediction precision, but also in the discussion of relationship between electricity consumption and the related factors. More electricity will be consumed as the population size increases. To reduce the electricity consumption, it is necessary to control the total population size and conserve the electricity resources. Like the $\operatorname{GMC}(1,2)$ and multiple linear regression, to make an electricity consumption prediction for the time period $t$, we need the population of the same period $t$. In this paper, to demonstrate the prediction performance, the actual population value from 2013 to 2015 is used. In theory, to make an electricity consumption prediction for the time period $t$, we can not know the population of the same period $t$. We can use the assumed value (default) for the population in the same period $t$. We can also consult the population experts on the future population value. As we all known, second-child policy has be carried out in Shandong Province. The population growth will speed up. The electricity consumption of Shandong Province will increase rapidly. The Shandong Province may face an ongoing electricity crisis which may be caused by the second-child policy.

For training data, the RMSPEPR of $\operatorname{GM}(1,1)$ model is 3.86 , that of traditional $\operatorname{GMC}(1,1)$ model is 1.80 , and that of $\operatorname{GMC}^{\frac{p}{q}}(1,2)$ is $1.67 . \operatorname{GMC}^{\frac{p}{q}}(1,2)$ is best. For the out-of-sample data, the RMSPEPO of $\operatorname{GM}(1,1)$ model is 7.64 , that of traditional GMC $(1,1)$ model is 6.76 , and that of $\operatorname{GMC}^{\frac{p}{q}}(1,2)$ is $5.91 . \operatorname{GMC}^{\frac{p}{q}}(1,2)$ is also best. Therefore, it may be used for other real cases for 
energy consumption forecasting. In theory, the grey forecasting model is suitable for addressing the limited sample forecasting problems [47]. Limited sample is suitable for short-term projection. In practice, the trends of these relative factors may change or the relationship between the reference series and comparison series may vary in the long term, so the $\operatorname{GMC}^{\frac{p}{q}}(1, \mathrm{~N})$ is also applicable for short-term projection.

From the perspective of theory contribution, the fractional order accumulation is extended to the multi-variable grey forecasting model and PSO is used to determine the optimal order. $\operatorname{GM}(1,1)$ model with fractional order accumulation is a particular form of the $\operatorname{GMC}^{\frac{p}{q}}(1, \mathrm{~N})$ model. With respect to $\operatorname{GM}(1,1)$ model with fractional order accumulation, the $\operatorname{GMC}^{\frac{p}{q}}(1, \mathrm{~N})$ model fully considers the effect of the relevant factors on the system, and it is a typical causal forecasting model. It can make full use of the information contained in the associated series of the predicted date. The first pair of original data by the model is effectiveness. Therefore, in the view of the available additional information, $\operatorname{GMC}^{\frac{p}{q}}(1, \mathrm{n})$ has better performance.

In future studies, it is suggested that the fractional order accumulation can be used in other models. Further, a goal is to set a threshold criterion for the relational analysis used to exclude certain parameters. The other variables can also be selected as the independent variables. The relationship between the other variables and the electricity consumption should be analyzed.

\section{Conflict of Interests}

The authors declare that they have no competing interests in this paper.

\section{Acknowledgements}

The relevant researches done in this paper are supported by the Leverhulme Trust International Network (IN-2014-020), National Natural Science Foundation of China (71401051), the Key Scientific Research Project of Colleges and Universities in Henan Province (17A630019) and the project of high-level talent in Hebei province (A2017003100). 


\section{References}

[1] F. E. Boran. Forecasting natural gas consumption in Turkey using grey prediction. Energy Sources, Part B: Economics, Planning, and Policy 2015,10(2): 208-213.

[2] Chai J, Guo J, Lu H. Forecasting energy demand of China using Bayesian Combination model. China Population, Resources and Environment 2008;18(4):50-55.

[3] Ji L Q. Forecasting petroleum consumption in China: comparison of three model. Journal of the Energy Institute 2011;84(1):34-37.

[4] Meng M, Niu D X. Annual electricity consumption analysis and forecasting of China based on few observations methods. Energy Conversion and Management 2011;52:953-957.

[5] Zhang M, Mu H L, Li G, Ning Y D. Forecasting the transport energy demand based on PISR method in China. Energy 2009;34: 1396-1400.

[6] Hongze Li, Sen Guo, Huiru Zhao, Chenbo Su and Bao Wang. Annual electric load forecasting by a least squares support vector machine with a fruit fly optimization algorithm. Energies 2012, 5(11):4430-4445.

[7] Zhu S L, Wang J Z, Zhao W G, et al. A seasonal hybrid procedure for electricity demand forecasting in China. Applied Energy 2011;88(11): 3807-3815.

[8] Li D C, Chang C J, Chen C C, et al. Forecasting short-term electricity consumption using the adaptive grey-based approach-An Asian case. Omega 2012;40(6):767-773.

[9] Yi-Chung Hu,Peng Jiang. Forecasting energy demand using neural-network-based grey residual modification models, Journal of the Operational Research Society. 2017,68:556-565.

[10] Zhaoshuang He, Caihong Li, Yulin Shen, et al. A hybrid model equipped with the minimum cycle decomposition concept for short-term forecasting of electrical load time series, Neural Process Letters, 2017,46(3):1059-1081. 
[11] Xin Ma, Zhibin Liu. Application of a novel time-delayed polynomial grey model to predict the natural gas consumption in China, Journal of Computational and Applied Mathematics, 2017, 324: 17-24.

[12] Sang-Bing Tsai, Youzhi Xue, Jianyu Zhang, et al. Models for forecasting growth trends in renewable energy, Renewable and Sustainable Energy Reviews, 2017,77: 1169-1178.

[13] Ning Xu, Yaoguo Dang, Yande Gong. Novel grey prediction model with nonlinear optimized time response method for forecasting of electricity consumption in China, Energy 2017, 118:473-480.

[14] Faheemullah Shaikh, Qiang Ji, Pervez Hameed Shaikh, et al. Forecasting China's natural gas demand based on optimised nonlinear grey models, Energy 2017, 140: 941-951.

[15] Abdulkerim Karaaslan, Mesliha Gezen. Forecasting of Turkey's sectoral energy demand by using fuzzy grey regression model,International Journal of Energy Economics and Policy, 2017, 7(1):67-77.

[16] Erick Meirade Oliveira, Fernando Luiz Cyrino Oliveira. Forecasting mid-long term electric energy consumption through bagging ARIMA and exponential smoothing methods. Energy, 2018, 144: 776-788.

[17] Hongye Guo, Qixin Chen, Qing Xia, et al. A monthly electricity consumption forecasting method based on vector error correction model and self-adaptive screening method. International Journal of Electrical Power \& Energy Systems,2018,95: 427-439.

[18] Zhen Shao,Chao Fu, Shan-Lin Yang, et al. A review of the decomposition methodology for extracting and identifying the fluctuation characteristics in electricity demand forecasting, Renewable and Sustainable Energy Reviews, 2017, 75: 123-136.

[19] Ergun Yukseltan,Ahmet Yucekaya, Ayse Humeyra Bilge. Forecasting electricity demand for Turkey: Modeling periodic variations and demand segregation. Applied Energy, 2017, 193:287-296. 
[20] Felipe L.C. Silva, Reinaldo C. Souza, Fernando L. Cyrino Oliveira, et al. A bottomup methodology for long term electricity consumption forecasting of an industrial sectorApplication to pulp and paper sector in Brazil. Energy, 2018, 144: 1107-1118.

[21] Aowabin Rahman,Vivek Srikumar, Amanda D.Smith. Predicting electricity consumption for commercial and residential buildings using deep recurrent neural networks, Applied Energy, 2018, 212: $372-385$.

[22] Kumar U, Jain V K. Time series model (Grey-Markov,Grey model with rolling mechanism and singular spetrum analysis) to forecast energy consumption in India. Energy 2010; 35(4):1709-1716.

[23] Zhou P, Ang B W, Poh K L. A trigonometric grey prediction approach to forecasting electricity demand. Energy 2006,31(14):2839-2847.

[24] Lee Y S, Tong L I. Forecasting energy consumption using a grey model improved by incorporating genetic programming. Energy Conversion and Management 2011;52(3):147-152.

[25] Akay D, Atak M. Grey predicton with rolling mechanism for electricity demand of Turkey. Energy 2007;32(9):1670-1675.

[26] Pao H T, Tsai C M. Modeling and forecasting the $\mathrm{co}_{2}$ emissions, energy comsumption, and economic growth in Brazil. Energy 2011;36(5):2450-2458.

[27] Pao H T, Fu H C, Tseng C L. Forecasting of $\mathrm{co}_{2}$ emissions, energy consumption and economic growth in China using an improved grey model. Energy 2012;40(1): 400-409.

[28] Li G D, Wang C H, Shiro Masuda, et al. A research on short term load forecasting problem applying improved grey dynamic model. International Journal of Electrical Power \& Energy Systems 2011;33(4),809-816.

[29] Iman Ghalehkhondabi, Ehsan Ardjmand, Gary R. Weckman, et al. An overview of energy demand forecasting methods published in 2005-2015. Energy Systems 2017,(8):411-447.

[30] Bo Zeng, ChengmingLuo, SifengLiu, et al. Development of an optimization method for the GM(1,N)model, Engineering Applications of Artificial Intelligence 55(2016):353-362. 
[31] Zheng-Xin Wang, Peng Hao. An improved grey multivariable model for predicting industrial energy consumption in China, Applied Mathematical Modelling 40 (2016):5745-5758.

[32] Tzu-Li Tien. The indirect measurement of tensile strength of material by the grey prediction model GMC(1,n). Measurement Science and Technology, 2005,16(6):1322-1328.

[33] Tzu-Li Tien. A research on the grey prediction model $\operatorname{GM}(1, n)$. Applied Mathematics and Computation, 2012,218:4903-4916.

[34] Zheng-Xin Wang, Peng Hao. An improved grey multivariable model for predicting industrial energy consumption in China. Applied Mathematical Modelling 2009; 33(8):3498-3510.

[35] Wu L F, Liu S F, Yao L G, et al. Grey system model with the fractional order accumulation. Communications in Nonlinear Science and Numerical Simulation 2013;18(7):1775-1785.

[36] Tzu-Li Tien. The indirect measurement of tensile strength by the new model FGMC $(1, n)$. Measurement 44 (2011):1884-1897.

[37] Xin Ma, Zhi-bin liu. Research on the novel recursive discrete multivariate grey prediction model and its applications. Applied Mathematical Modelling 2016;40:4876-4890.

[38] Zheng-Xin Wang. Multivariable time-delayed of $\operatorname{GM}(1, N)$ model and its application. Control and Decision 2015,30(12):2298-2304.

[39] Wu L F, Wang Y N, Liu S F. Grey convex relation and its properties prediction. System Engineering Theory \& Practice 2012;(32)7:1501-1505.

[40] Kennedy J, Eberhart R. Particle swarm optimization. Proceedings of IEEE International Conference on Neural Network 1995;1942-1948.

[41] Karl Mason, Jim Duggan, Enda Howley. Multi-objective dynamic economic emission dispatch using particle swarm optimisation variants. Neurocomputing 2017;(27):188-197.

[42] Hwang Ho Kim, Do Gyun Kim, Jin Young Choi, et.al. Tire mixing process scheduling using particle swarm optimization. Computers \& Industrial Engineering 2017;(110):333-343. 
[43] Karl Mason, Jim Duggan, Enda Howley. A meta optimisation analysis of particle swarm optimisation velocity update equations for watershed management learning. Applied Soft Computing, 2018,62: 148-161.

[44] Ke Chen, Fengyu Zhou, Aling Liu, Chaotic dynamic weight particle swarm optimization for numerical function optimization. Knowledge-Based Systems 2018,139:23-40.

[45] Wenxing Ye, Weiying Feng, Suohai Fan. A novel multi-swarm particle swarm optimization with dynamic learning strategy. Applied Soft Computing 2017;(61) :832-843.

[46] Mustafa Servet Kiran. Particle swarm optimization with a new update mechanism.Applied Soft Computing 2017;(60):670-678.

[47] Wu L F, Liu S F, Yao L G, et al. The effect of sample size on the grey system model. Applied Mathematical Modelling 2013;37(9):6577-6583. 